\title{
Computational Schemes in the Design of Novel Materials for Energy Savings
}

\author{
Ohunene Hafsa Aliyu* \\ Department of Building, Federal University Birnin Kebbi, P.M.B 1157, Kebbi State, Nigeria.
}

ABSTRACT: The current demand for lightweight energy efficient materials in industries to help in addressing the current challenges faced in reducing green house gas emissions is one of the motivations behind innovative material design. This paper has tried to review in particular the importance of the hierarchical multiscale modelling strategy in the design of novel lightweight materials. It also highlights on one of the hierarchical multiscale modelling methodologies from ab-initio level to macro level in predicting macroscopic material behaviour and the impact this simulation strategy will have on the development of innovative materials. It highlights on the limitations of this modelling strategy such as: the unreasonable computational time associated with the relaxation of polymeric chains or entanglements as well as scale bridging approaches between low level and high level models which are areas of current research interest.

KEYWORDS: Multiscale modelling, lightweight materials, energy-efficiency, scale bridging, length scale.

[Received February 6, 2019, Revised September 25, 2019, Accepted November 10, 2019] Print ISSN: 0189-9546 | Online ISSN: 2437-2110

\section{INTRODUCTION}

The building blocks of natural biological materials are based on nano-composites with complex hierarchical arrangement. As a result of the hierarchical structure of these biological materials, they tend to exhibit outstanding stiffness; toughness and strength at their low concentrations. Although, the mechanical behaviour of their underlying constituents is rather moderate (Nikolov et al., 2010). It is the relationship that exists between the hierarchical structural arrangement of these natural biological materials and the mechanical behaviour of the constituents that has been a source of motivation (among many others) behind the design of novel materials by both engineers and material scientists alike. This motivation has inspired the design of novel materials to mimic the component, structure as well as systems existing in natural biological materials by adopting the recently developed nanotechnology which aids design at micro and nano scale.

At these scales, the hierarchical organization as well as the flawless combination of the complex material structure can be realized which are then targeted to enhance the material properties such as: improved strength and toughness, high stiffness and high damping, improved thermal conductivity and electrical permittivity, improved permeability weight reduction etcetera. This eventually improves materials performance when employed in particular industrial applications (Ashby et al., 1995;
Kanoute et al., 2009; Yao et al., 2010). Scholars such as Fratzl et al. (2004), Meyers et al. (2008) and Espinosa et al. (2009) have all studied how the arrangement of the hierarchical structure of natural biological material relates to its enhanced mechanical behaviour.

\section{NOVEL LIGHTWEIGHT MATERIALS}

The global challenges faced in reducing green house gas emissions across the building, transportation and industrial sector necessitate the need for adopting energy efficient systems aimed at saving fossil fuel and production cost (Mambo, 2017). Energy efficiency can however, be achieved through the adoption of a number of advancements in technology. One of which is the weight reduction in materials (Joost, 2012).

Hence, the current rise in demand for novel lightweight materials which are not only cost effective but also have high performance. The current trend of activities involved in the design of novel lightweight materials encompassing material creation to end product is shown schematically in Figure 1. 

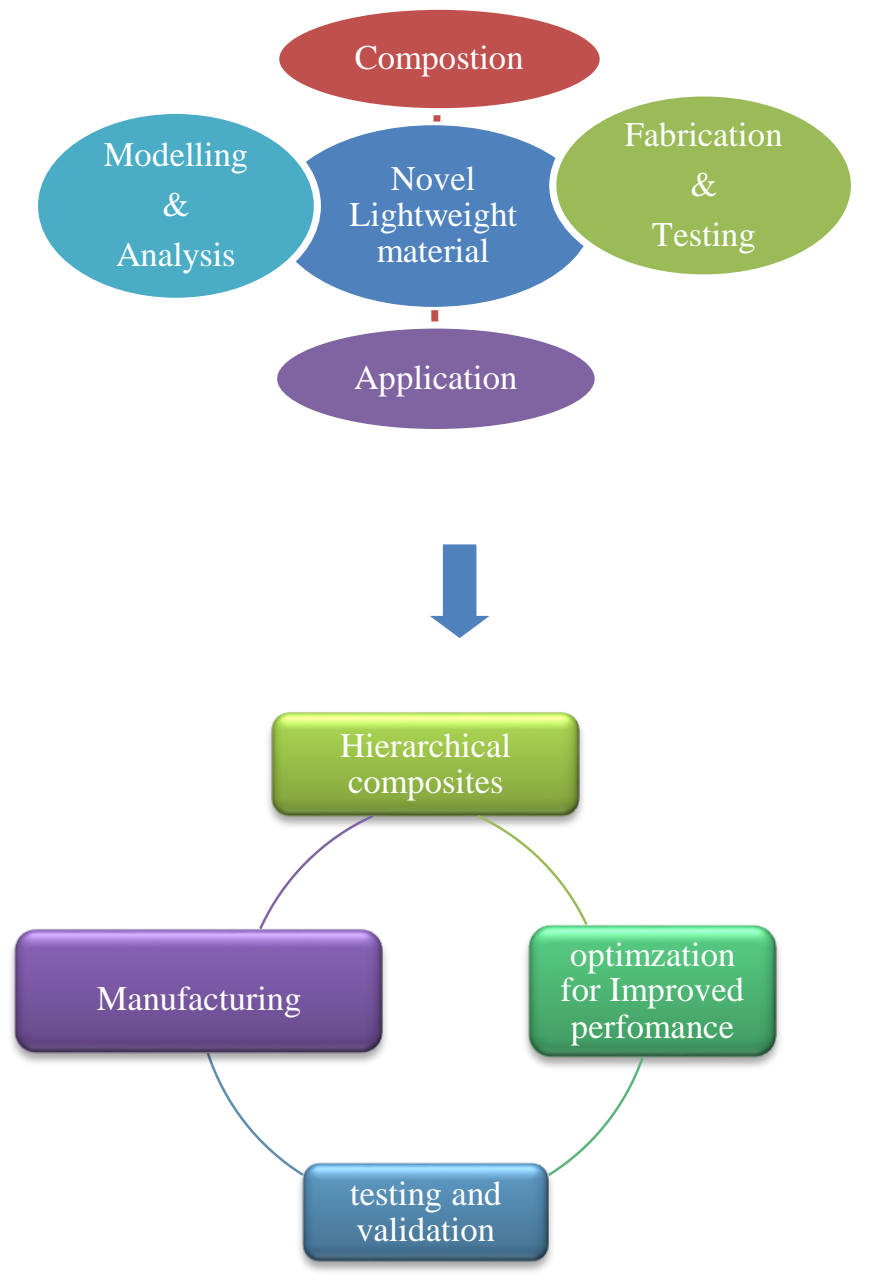

Figure 1: Modern trend for development of novel materials adapted from Ahmed and Azoti, 2015.

Examples of such lightweight materials include high strength steel, magnesium alloys, aluminium alloys, amorphous polymers, polymer composites (for example, graphene based polymer composites), carbon fibre, etcetera (Ahmed and Azoti, 2015). Since weight reduction is key in ensuring energy efficiency of materials, facilitating their design to match current demand, requires carrying out research in the fields of material science and computational micromechanics to deal with the likely challenges to be faced with safety, prediction, manufacturing cost, modelling to determine material behaviour as well as improved performance (Bouvard et al., 2009; Joost, 2012).

For example, assessing the instability of lightweight materials under thermal and mechanical loading which eventually results in catastrophic material failure (such as: cracking, creep, brittle failure etcetera) as well as studying the energy absorption behaviour of the lightweight materials in deforming under impact loads (that is at high strain rates)
(Aliyu, 2019). These failure mechanism, are considered the major shortcomings of most novel materials and are usually controlled by grain boundaries (Stevens, 2001; Kassnor, 2008; Sheikh-Ali, 2016). So that it is the properties of these grain boundaries (mobility, sliding rate, diffusion and so on) which moderate the causes of material failure that are of interest to material scientists and engineers alike. These properties tend to vary over several orders of magnitude (Tumbull and Hoffman, 1954; Aust et al., 1994).

As such, an efficient modelling strategy is vital for thorough understanding of the behaviour of materials (from electron to macroscopic level) when subjected to various environmental and loading conditions as well as manufacturing processes. This can however, be achieved either experimentally (for example, crashworthiness, material testing, etcetera) or via computational modelling methods (Kouznetsova et al., cited in Galvanetto and Aliabadi, 2010). However, because of the time and expenses involved with experimental testing, computational method is preferred as it is more cost effective and able to predict materials behaviour at a level of detail and timescale which is not experimentally feasible (Pablo and Curtain, 2007; Bouvard et al., 2009).

To this end, the hierarchical multiscale modelling strategy will be discussed as this modelling strategy can be adopted in understanding the behaviour of materials from its ab-initio level (electron structure level) to the continuum mechanics level (macroscopic level).

\section{A. Computational Method}

Present-day computational approach employed in the analysis and design of structural materials and components have proven to be an invaluable aid particularly with the challenges faced in the prediction of novel material behaviour as well as optimization of structural material for use in certain industrial applications. This novel approach incorporates micro-macro structure property relationship within a computational scheme with the hopes of manipulating the material microstructure aimed at influencing the macroscopic mechanical behaviour of the (structural) material to attain a desired outcome in material behaviour (Bouvard et al., 2009). The methodology reviewed in this paper which incorporates this structure property relationship in predicting the mechanical behaviour of structural materials in a simulationbased design frame work from ab-initio to continuum mechanics level, is the hierarchical multiscale modelling scheme. This modelling scheme bridges length scales from nano-macro (that is quantum, molecular and continuum) through the establishment of a set of structure property relationships as shown in Figure 2. 


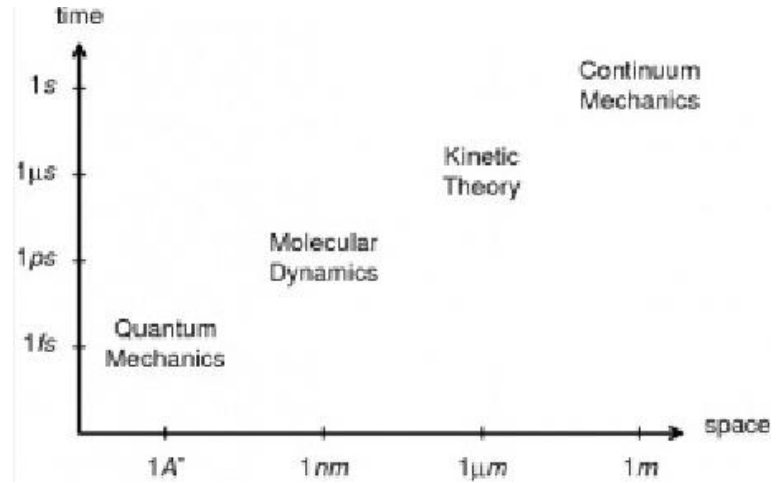

Figure 2: Illustration of the multi-physics hierarchy adapted from Weinan and Jianfeng, 2011.

\section{B. Adoption of computational modelling in novel material design}

With the emergence of new lightweight structural materials employed in certain industrial application, it is imperative to thoroughly understand the relationship that exists between the materials microstructure as well as the physics of its underlying molecular mechanics in order to be able to predict the material properties (over a wide range of temperature, strain rates and stress states) as well as its mechanical behaviour (Pablo and Curtain, 2007). An understanding of this relationship requires carrying out expensive mechanical testing under a wide variety of environmental and loading conditions. However, from an economical perspective performing straight such forward experimental measurements where the micro and molecular structure are explicitly considered is almost not feasible; hence the need for an efficient modelling strategy (Pablo and Curtain, 2007; Nguyen et al., 2012; Kouzentsova et al., 2010). Computational methods, especially multiscale modelling is one such modelling strategy which integrates

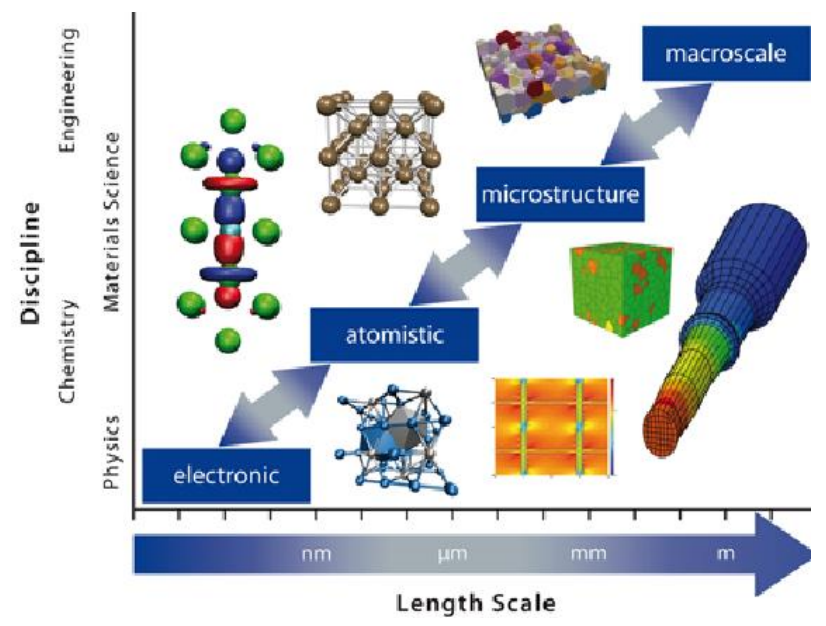

Figure 3: A seamless integration of models on different length scales into one coherent multiscale modelling frame work. material properties from the smallest length and time scales into the design process. This integration of material properties from models across multiple length and time scales are shown in Figures 3 and 4. This multiscale modelling strategy can be classified on the basis of problem formulation as (Matous et al., 2017):

- The concurrent method: for this method, multiple models (for example, micro and macro scale models) are concurrently addressed in real-time to give a seamless integration of information between models in a solo computer based simulation.

- The hierarchical method: runs discrete computer based simulations in computing the structure property relation at a particular length scale and the information obtained is passed to a higher scale using averaging theorems as shown in figure 4 . As such, the relationship between scales, are of a hierarchical nature.

- Hybrid method: this method gives useful information on the properties of different multiscale solvers such as: multigrid method, wavelet-based methods, generalized finite element method (Tadmor and Philips, 1996; Fish 2009; Miehe and Bayreuther, 2007; Bouvard et al., 2009; Plews and Duarte, 2014; Matous et al., 2017).

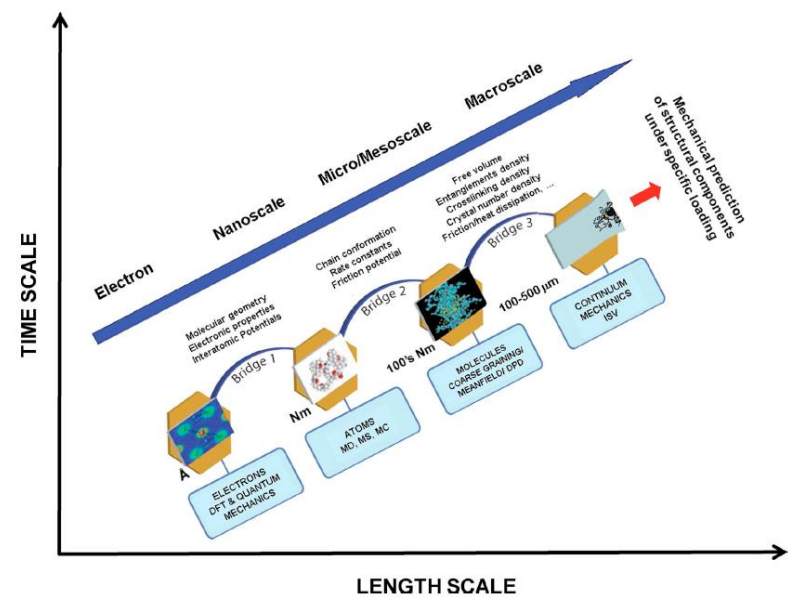

Figure 4: Scheme of a general hierarchical modelling approach for polymers adapted from Bouvard et al. 2009.

Taking the case of metals as an example, the hierarchical multiscale strategy comprises energy potentials (established from quantum mechanics) employed in the atomistic simulations to examine mobility and interactions as well as dislocation nucleation; so that for the development of polycrystalline plasticity work hardening model, atomistic simulations are carried out. The information obtained from the atomistic simulation is used in discontinuous dislocation dynamics to examine the dislocation network as well as structure development for work hardening which is eventually used at the continuum level (that is macro-scale) for analysis at the component scale level where structural elements are 
considered. See Figure 2 for illustration of the hierarchical multiscale strategy (Bouvard et al., 2009).

For amorphous polymers, hierarchical modelling is still in its early stages. The wide scope in length and time scales associated with amorphous polymers complicates the mulitscale modelling process. Despite the complication of this technique, it is still very crucial in simulating the behaviour of these materials (Flory, 1989; Binder, 1995; Bouvard et al., 2009).

Traditional molecular dynamics, simulations were limited to time-scales of few picoseconds which were not sufficient enough as it gave many pieces of information which led to wrong conclusions. This is because for simulations to investigate thoroughly the properties of grain boundaries which mediate against material failure, a wider range of length and time scale is needed. Modern simulation methods now cover a much wider range of length and time scales. This however creates a paradox, because as the length and times scales are increased, the accuracy of simulation is reduced. It is however hoped that by introducing molecular dynamics within the field of quantum mechanics accuracy will be increased as the simulation will be free of any empirical parameter inputs. A well known technique which implements the above method is the Car-Parrinello molecular dynamics (Car and Parrinello, 1985).

Another popular technique is the hybrid method which mixes quantum mechanics and molecular mechanics schemes (Warshel and Levitt, 1976; Field et al., 1990). The most fascinating of these schemes are those that go further beyond atomistic resolution to model nano-scale systems as well as other soft materials (Espanol, 1995). With the current advancement in simulation technology allowing system description at quantum mechanics, molecular mechanics as well as mesoscopic level, the major drawback is that accuracy has progressively decreased as the length and time scales increased (Lyubartsev et al., 2009). Now beyond atomistic resolutions, simulation models are generally as a result of coarse graining.

Coarse grain models help to bridge the size and time gap between computational and experimental simulations by reducing the number of degrees of freedom considered which allows an increase in simulation speed and length scale. There is presently no specific way of achieving coarse graining with off-lattice framework; for heterogeneous systems as in material design; finite element and grid-based models are normally used (Groot 2000; Marrink et al., 2004; Bouvard et al., 2009).

\section{HIERARCHICAL MODELLING}

Modelling physical systems at various levels of detail (that is at different length scales) results in a hierarchy of models. For such hierarchical systems, models at the lower level are a refinement of those at the higher level. For example, in modelling the dynamics of gases; depending on the level of detail desired, the following hierarchical order may be followed that is modelling from quantum mechanics level to molecular dynamics level to kinetic theory level and finally continuum mechanics level. The models at the higher level (continuum mechanics level) are considered the most basic and only able to model properties such as: macroscopic density, velocity, temperature, etcetera; models at the lower level (quantum mechanics level) are more refined and detailed (Weinan, 2011).

\section{A. Hierarchical Multiscale Modelling Methodology}

Hierarchical multiscale modelling is aimed at performing detailed simulations from first principle with the intention of obtaining full comprehensive information about a system. It shows how the results obtained from a more accurate and detailed fundamental model (for example, electronic structure level) is transferred to a much basic higher model (for example, continuum level) this has been illustrated schematically in figure 5 (Lyubartsev et al., 2004).

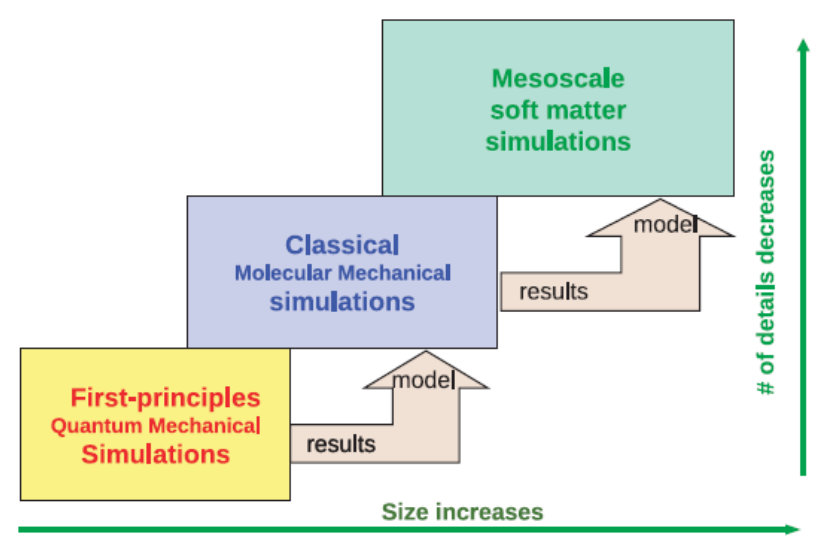

Figure 5: Schematic representation of hierarchical multiscale approach: results of simulation of a more detailed model are used to build a model for simulation on a larger scale adapted from Lyubartsev et al. 2009.

\section{B. A general approach to hierarchical multiscale modelling}

As mentioned earlier, a cost effective and time efficient computational approach for modelling beyond the atomistic level (that is at the molecular level) is by use of well structured coarse grain model potential energy functions. These models tend to focus on the degrees of freedom of utmost importance and are able to describe the equilibrium structural properties of interest.

The simulation of these coarse grain models to determine the equilibrium properties can either be by:

- Hamiltonian molecular dynamics: although, simulations of this nature do not exhibit accurate dynamic behaviour or

- Lavevian or Brownian dynamics: while this method gives an accurate dynamic behaviour, it may not be always be appropriate as the method depends on the 
make-up of the system as well as the degree of coarse graining or the

- $\quad$ Monte Carlo method (Theodorou, 2004; Underhill and Doyle, 2004; Davtyan et al., 2015).

Similarly, building effective coarse grained potential can be achieved either by using radial distribution functions or force matching approach (Izvekov et al., 2004). With the force matching approach, linking the ab-initio with the atomistic molecular level is quite straight forward. However, for the next level between the atomistic and coarse grained levels, the multiscale modelling becomes quite complicated as quite a number of additional hypotheses will have to be made, which is the Achilles heels of this approach in terms of hierarchical multiscale modelling strategy (Lyubartsev et al., 2004).

\section{Multiscale modelling strategy for evaluating the effective coarse grain potentials using radial distribution functions}

The procedure for this strategy is as described in this section. Firstly, a detailed simulation is carried out from the underlying ab-initio level (electronic structure level) which gives full information about the system. Based on the full information obtained, it becomes possible to establish a coarse grain model; radial distribution functions obtained from the detailed simulation can be used to link positions with interesting degrees of freedom which are worthy of noting. Next we try to determine from the observed outcome of (that is from the radial distribution function) the likely interaction potential within the coarse grain model which matches exactly the outcome observed from the radial distribution function using the full detailed information obtained. To achieve this, we need to solve the inverse problem which entails the reconstruction of the interaction potential from the outcome obtained earlier from the radial distribution function (Lyubartsev et al., 2009).

Obtaining the effective interaction potential from a solution of the inverse problem using the inverse Monte Carlo method, Lyubartsev and Laaksonen, (1995), enables us to enhance our length scale such that the evaluated effective interaction potential can be used for simulation at a much higher scale of resolution (Lyubarstev et al., 2009; Dunn and Shuits, 2012). It is clear that obtaining a solution of the inverse problem is crucial. This implies that a solution must exist and must be to the only one of its kind (that is it must be unique). To this end, the solution is considered unique in terms of pairwise potentials and radial distribution function provided that no attempt is made to consider all other possible potentials. As a consideration of all other possible potentials not only removes the uniqueness of the solution as it increases the number of degrees of freedom considered, which slows down the computational speed (Shish et al., 2006; Shish et al., 2007; Lyubarstev et al., 2009; Dunn and Shuits, 2012; Izovekov and Voth, 2006).

\section{SUMMARY}

Molecular dynamic simulations used at the molecular level to model deformation mechanics are currently limited to a time scale of a few nanoseconds. For materials such as polymers having entanglements, the relaxation time is likely to be quite high and may become computationally unreasonable. So that while coarse-graining method employed in molecular dynamics is able to extend our length scales, the problem of time scaling is still inherent with molecular dynamic models. Similarly, the passing of information using structure-property relation from a lower level to a much higher level (for example, from atomistic level to continuum level), is also constrained due to the limited amount of time inherent in molecular dynamic model which hinders it from capturing all the deformation mechanics which controls properties such as viscoelasticity/plasticity of materials. This suggests that some important micromechanical information's are still unclear.

Scholars such as Horstemeyer, 2001; Horstemeyer and Wang, 2003; from their study showed the importance of multiscale modelling in metals, while Theodorou (2004 and 2007); Bouvard et al. (2009); who reviewed the hierarchical multiscale modelling of polymers, emphasised on the impact multiscale modelling could have on optimization of polymer morphologies for specific industrial use.

\section{CONCLUSION}

Designing to meet the current demand for novel lightweight materials that satisfy the requirements of energy savings as well as a whole range of other complex industrial application is made possible by thoroughly understanding the properties as well as the relationships that exists within internal material microstructure (that is the microstructure property relation). This understanding assists in evaluating the macroscopic mechanical properties such as: strength, toughness, and so on. This is usually achieved by adopting computational modelling schemes (for example, multiscale modelling) which incorporates micro-macro structure property relationships. This modelling method provides a cost-effective and efficient strategy for simulating, enhancing as well as predicting behaviour of novel materials at a level of detail and time scale which is not feasible experimentally.

This is evident in the atomic simulation of interfaces under a wide range of environmental and loading conditions (which influences strength and deformation). It provides the detailed information needed to set-up predictive physical based models employed in simulating microstructure deformation, evolution as well as fracture which aids in identifying microstructures with enhanced mechanical properties. Adopting this modelling strategy in designing novel lightweight materials for industry will significantly reduce energy consumption ensuring a much greener environment. Since the building and transportation sector contributes to over $55 \%$ of the global energy consumption 
which involves the combustion of fossil fuel that releases operational carbon $\left(\mathrm{CO}_{2}\right)$ which contributes significantly to the overall carbon footprint. This highlights the importance of computational modelling strategy in designing lightweight materials for energy savings.

\section{REFERENCES}

Aliyu, O. H. (2019). Design Recommendations for Steel Beams to Prevent Brittle Fracture. Nigerian Journal of Technologycal Development, 16(2): 78-84.

Ashby, M. F.; L. J. Gibson, U. West, and R. Olive. (1995). The Mechanical Properties of Natural Materials. Material Property Charts. Proc. R.Soc. A 450: 123-140.

Aust, K.T.; U. Erb, and G. Palumbo. (1994). Interface control or resistance to intergranular cracking. Materials Science and Engineering, A 176 (1-2): 329-334.

Binder, K. (1995). Monte Carlo and Molecular Dynamics Simulations in Polymer Science. New York: Oxford University Press.

Bouvard, J. L.; D. K. Ward, D. Hossain, S. Nouranian, E. B. Marin, M.F. Horstemeyer. (2009). Review of Hierarchical Multiscale Modelling to Describe the Mechanical Behavioir of Amorpous Polymers. Journal of Engineering Materials and Technology, 131.

Car, R. and Parrinello, M. (1985). "Unified Approach for Molecular Dyanmics and Density Functional Theory". The American Physics Societ, 55(22) :2471-2474.

Davtyan, A.; J. F. Dama, G. A. Voth, and H. C. Anderson. (2015). Dynamic force matching: A method for construction dynamic coarse-grained models with realistic time dependence. Journal of Chemical Physics , 142; doi: 10.1063/1.4917454.

De Pablo, J. J. and Curtain, W. A. (2007). Multiscale Modelling in Advanced Materials Research. MRS Bull, 32(11): 905-911.

Dunn, W. L. Shultis, J. K. (2012). Exploring Monte Carlo Methods. San. Diego U.S.A : Elsevier.

Engquist, W. E. B.; X. Lao, and W Ren, (2007). Heterogenous Multiscale methods: a review. Commun. Comput. Phys., 2: 367-450.

Espinosa, H. D.; J. E. Rim, F. Barthelat, and M. J. Buehler. (2009). Merger of structure and material in nacre and bone-pespective on denow biomimetic materials. Progress in Material Science, 54 :1059-1100.

Elmarakbi, A. and Azoti, W. L. (2015). Novel Composite Materials for Automative Applications: Concepts and Chanllenges for Energy-Efficient and Safe Vehicles. 10th International Conference on Composite Science and Technology ICCT/10.

Flory, P. (1989). Statistical Mechanics of Chain Molecules Interscience. New York.

Field, M. J.; P. A. Bash, M. Karplus. (1990). A combined qunatum mechanical and molecular potential for molecular dynamics simulation. Jornal of Compuational Chemistry, 11(6): 700-733.

Fish, J. (2006). Bridging the scales in nano engineering and science. J. Nanopart. Res., 8: 557-594.
Fish, J. (2009). Multiscale Methods: Briding the Scales in Science and Engineernig. U.S.A: Oxford University Press.

Fratzl, P.; H. S. Gupta, E.P. Paschalis, and P. Roschger. (2004). Structure and Mechanical Quality of the Collagen-mineral Nano-composite in Bone. Journal of Material Chemistry, (14): 2115-2123.

Galvanetto, U. and Aliabadi, M. H. (2010). Multiscale Modelling in Solid Mechanics. 3: 1-42 Imperial College Press 57 Covent Garden London.

Groot, R. (2000). Mesocopic Simulation of Polymersurfactant Aggregation. 16(19): 7493-7502.

Horstemeyer, M. F. and Wang, P. (2003). Cradle-toGrave Simulations Based Design Incoporating Multiscale Microstructure-Property Modelling: Rein Vigorating Design with Science. J. Comput. Aided. Mater. Des., 10: 13-34.

Izvekov, S. and Voth, G. A. (2006). Modelling real dynamic in the coarse-grained representaion of condensed phase systems. J. Chem. Phys, 125(15): 151101

Joost, W. (2012). Reducing vehicle weight and imporving U.S. energy efficiency using integratd compuational materials engieering. JOM, 64(9): 1032-1038.

Kanoute, P.; P. D. Boso, J. L. Chaboche, B. A. Schreller. (2009). Multiscale Methods for Composites: A Review. Arch Comput Methods Eng, 16: 31-75.

Kassnr, M. E. (2008). Fundermentals of Creep in Metal and Alloys $2^{\text {nd }}$ Ed. Science Direct Elsevier: 221-246.

Kouznetsova, V.G.; M. G. D. Geers, and W. A. M. Brekelmans. (2010). Muliti-scale compuational homogenization: Trends and Challenges. Journal of Compuational and Applied Mathematics, 234(7): 2175-2182.

Kremer, K. and Muller-Plathe, F. (2001). Multiscale Probles in Polymer Science: Simulation and approaches. MRS Bull , 26(3): 205-210.

Lybartsev, A.; Y. Tu, and A. Laaksonen. (2009). Hierarchical Multiscale Modelling Scheme from First Principle to Mesoscale. Journal of Comuptational and Theoretical Nano Science, 6: 1-9.

Mambo, A. D.; O. H Aliyu, and A. K. Evans. (2017). Automation and Optimization of Lighting for Energy Savings: A case study of a Faculty Boardroom. Nigerian Journal of Renewable Energy, 17(1\&2): 37-42

Marrink, S. J.; A. H. De Vres, and A. E. Mark. (2004). Coarse grained model for semi quantitative lipid Simulation. Journal of Physical Chemistry D., 108(2): 750-760.

Matous, K.; M. G. O. Geere, V. G. Kouznetsova, and A. Gillman. (2017). A review of predictive nonlinear theories of multiscale modelling of heterogenous materials. Journal of Computational Physics, 330: 192-220.

Meyers, M. A.; P.-Y. Chen, A.Y.-M Lin, and J. Muyco. (2008). Biological Materials: Structure and Mechinical Properties. Progress in Material Science, N0.1: 1206.

Miehe, C. and Bayreuther, C. (2007). On Multiscale FE analysis of Heterogenous Structures: From Homogenizatiion to Multi Solvers. Int. J. Numer. Methods Eng., 71: 1135-1180.

Nguyen, V.P.; M. Stroven, Sluys, L.J. (2012). Mulitiscale continous and disconinous modelling of heterogeneous mateials: A review on recent developments. Elsevier. 
Plews, J. and Duarte, C. (2014). Bridging Multiple Structural Scales with a Generalized Finite Element Mehthod. Int. J. Numer. Methods. Eng., :180-201.

Sheikh-Ali, A. D. (2016). Encyclopedia of Materials: Science and Technology $2^{\text {nd }}$ Ed. Science Direct Elsevier: 3624-3625

Shih, A.Y.; P. L Freddolino, A. Arkhipov, and K. Schullen. (2007). Assembly of lipoprotein particles revealed by caorse-grained molecular dynamics simulations. Jorunal of Structural Biology,: 579-592.

Shih, A. Y.; A. Arkhipov, P. L. Freddolino, and K. Schullen. (2006). Coarse-grained protein-lipid model with application to lipo protein particles. Journal of Physical Chemistry B, 110: 3674-3684.

Stevens, R. N. (2001). Grain Boundary Sliding: Experimental Observations. Science Direct Elsevier: 36223624

Tamdor, E. (1996). Quasi Continuum Analysis of Defects in Solids. Philos. Magr A(73): 1529-1663.
Tumull, D. and Hoffman, R. E. (1954). The effect of relative crystal and bounday orientation on grain boundary diffusion rates. Acta Metallurgical , 2: 419-426.

Theodorou, D. (2004). Understanding and Predicting Structure-Property Relations in Ploymeric Materials Through Molecualr Simulations. Mol. Phys. ,: 147-166.

Theodorou, D. (2003). Understanding and Predicting Structure-Property Relations in Ploymeric Materials Through Molecular Simulaitons. Molecular Physics Foundations of Molecualr and Simulation FOMMS, 102(2 part 1): 147-166.

Underhill, P. T. and Doyle, P. S. (2004). On the CoarseGraining of Polymeric Materials Through Molecular Simulations. J. Non-Newtonian Fluid Mechanics , 122: 3-31.

Warshel, A. and Levitt, M. (1976). Theoretical Studies of Enzymic Reaction: Dielectric and Steric Stabilization of the Carbonium ion in the Reaction of Iysozyme. Journal of Molecualr Biology, 103(2): 227-249.

Weinan, E. (2011). Principles of Multiscale Modelling. UK: Cambridge University Press.

Yao, H.-B.; H.-Y. Fang, X.-H. Wang, and S.-H. Yu. (2012). Hierarchical assemby of micro -/nano-building blocks : bio-inspired rigid structuarl functional material. The Royal Society of Chemistry, 40: 3764-3785. 